\title{
Industrial Scalable Additives for Enhanced Decomposition of Plastic Waste Through Photocatalysis
}

\author{
Thomas W Colburn ${ }^{1}$, Todd J Toops ${ }^{1 *}$, and Andrew A Wereszczak ${ }^{2}$ \\ ${ }^{1}$ Applied Catalysis and Emissions Research Group, Oak Ridge National Laboratory, USA \\ ${ }^{2}$ Materials Science and Technology Division, Oak Ridge National Laboratory, USA
}

Submission: June 12, 2020; Published: June 24, 2020

*Corresponding author: Todd J Toops, Applied Catalysis and Emissions Research Group, Oak Ridge National Laboratory, Knoxville, USA

\begin{abstract}
Polymer pollution is a dangerous environmental problem that can lead to the spread of toxic chemicals and microplastics throughout aquatic biomes. Commonly littered plastic from consumer goods and products with a limited or single use can be embedded with photocatalysts to avoid an increase in the amount of plastic present in Earth's bodies of water. The photocatalyst nanoparticles, less than $100 \mathrm{~nm}$ particle size, were integrated into polyethylene terephthalate, high-density polyethylene, low-density polyethylene, and polystyrene and exposed under ultraviolet light over two independent experimental trials examining both the environmental factors impacting decay and the physical parameters required to implement this solution on a large scale. The plastics were chosen due to their prolific use in items such as grocery bags, bottles, and packaging. The first half of the project examined the effects of water and light frequency on polymer decay, and the second half of the project examined photodecomposition of polymer films. We report that low-density and high-density polyethylene films with titanium dioxide decayed approximately 11.7 and 9.6 faster than control samples, respectively. With an estimated price of less than half the cost of a shopping bag for titanium catalyst integration and a retention of the polymer's tensile strength with a $2 \mathrm{wt} \%$ catalyst concentration, photocatalytic decomposition is a feasible, scalable method for the prevention and future reduction of polymer waste from realistic products littered in the environment.

Keywords: Plastic litter, Photodegradation, Titanium dioxide, Polyethylene, Photocatalysis,
\end{abstract}

\section{Introduction}

Plastic pollution is a mounting global concern due to improper or an absence of waste management [1]. Polymers are a threat to animal life and the chemical stability of the environment, especially waterways. Erikson, et al. estimate there are 5.25 trillion plastic polymer fragments with a total weight of 268,940 tons [2]. The growth in the plastic industry along with a growing world population places a burden on the environment with higher amounts of plastic litter and more waste entering waterways. Moreover, plastic particles on the microscopic scale are capable of harboring waterborne contaminates and toxins due to a large particle surface area [1]. These microscopic particles of plastic are predicted to subsist for centuries in the environment without the ability to fully decompose [3]. The goal of this project was to investigate a potential solution to plastic pollution from litter using low-cost and environmentally benign additives resulting in accelerated decay with exposure to sunlight. Polyethylene terephthalate (PET), high-density polyethylene (HDPE), lowdensity polyethylene (LDPE), and polystyrene (PS) are four commonly used household plastics with environmental lifetimes of approximately 450, 400, 15, and 450 years, respectively [3]. Bottles and containers are commonly made of PET and HDPE, plastic bags are commonly made of LDPE, and PS is used in a variety of applications including packing materials [4]. The prolific and unabated use of these long-life polymers dictates a need to minimize the plastics' environmental impact when disposed.

The polymers examined possess varying modes of decomposition [5,6]. For example, HDPE and LDPE undergo chain scission, and subsequent chain unzipping, releasing gaseous ethylene and other alkenes and acids [5]. In contrast, PET and PS undergo main chain scission into solid-state products that eventually decay further into carbon monoxide, carbon dioxide, and oxygenates [5]. Single-use products such as plastic bags lack photostabilizers making these plastics more susceptible to photodecomposition compared to industrial or chemically stabilized plastics used in building materials and durable outdoor applications. This photodecomposition can be initiated, stimulated, or supported by the use of photocatalytic additives to the polymer. Previous studies have found that some catalysts 
and molecular structures can contribute to photodecomposition [7-10]. While those studies do focus on polymer decay with nanoparticle additives, the outlined study focuses on studying both environmental effects and degradation of films mimicking actual products such as plastic bags or milk jugs. This study elucidates the industrial scalability, economic feasibility, and processing limitations to develop a photocatalytically-active polymer production analog that both retains a primary function while decaying upon prolonged exposure to sunlight. Moreover, this study focuses especially on additives that are cost effective and do not compromise the tensile strength of the polymers in their intended functions.

The process of photocatalytic decomposition of plastics on the macroscopic and microscopic levels is illustrated in Figure 1. Various metal oxides and many nanoparticles exhibit photocatalytic properties due to their band gap energies and crystalline structures, which can be used to initiate the decomposition of hydrocarbons [7]. Band gaps less than $4 \mathrm{eV}$ and greater than $2 \mathrm{eV}$ were chosen to maximize absorption in the solar spectra while maintaining high-energy exciton pairs. Moreover, certain crystalline structures have enhanced photocatalytic effects due to band gap size and location modification. Upon exposure to ultraviolet (UV) light, the photocatalyst molecules release free electrons leaving electron holes. These free electrons cause the decomposition of polymers by a variety of mechanisms. Thus, the sun's UV and visible rays can be potentially employed to combat the widespread presence of plastics in the environment. Titanium dioxide $\left(\mathrm{TiO}_{2}\right)$, zinc oxide $(\mathrm{ZnO})$, tungsten trioxide $\left(\mathrm{WO}_{3}\right)$, and iron (III) oxide $\left(\mathrm{Fe}_{2} \mathrm{O}_{3}\right)$ are catalysts active in the sun's UV range and will be the focus of this study. Titanium dioxide, in particular, has attracted significant research focus due to the photocatalysts' activity range, band gap, abundance, and cost [11,12]. After the polymer laminate decomposes, the photocatalysts would interact with the environment necessitating environmentally benign materials. While the full environmental health effects of the catalysts used in experimentation are being investigated $[13,14]$, the $\mathrm{TiO}_{2}$, for example, has been reported to have some accumulation in mammalian cells [14]. Some catalysts, especially $\mathrm{TiO}_{2}$ nanoparticles, are currently used in or investigated for consumer products like sunscreen, house paint, as antimicrobial coatings [15], as a tool for environmental clean-up [16,17], and as FDA-approved food colorants [18]. Care must be taken to tailor the particle's size, processing techniques, and manufacturing parameters to ensure safe introduction into the polymer matrix.

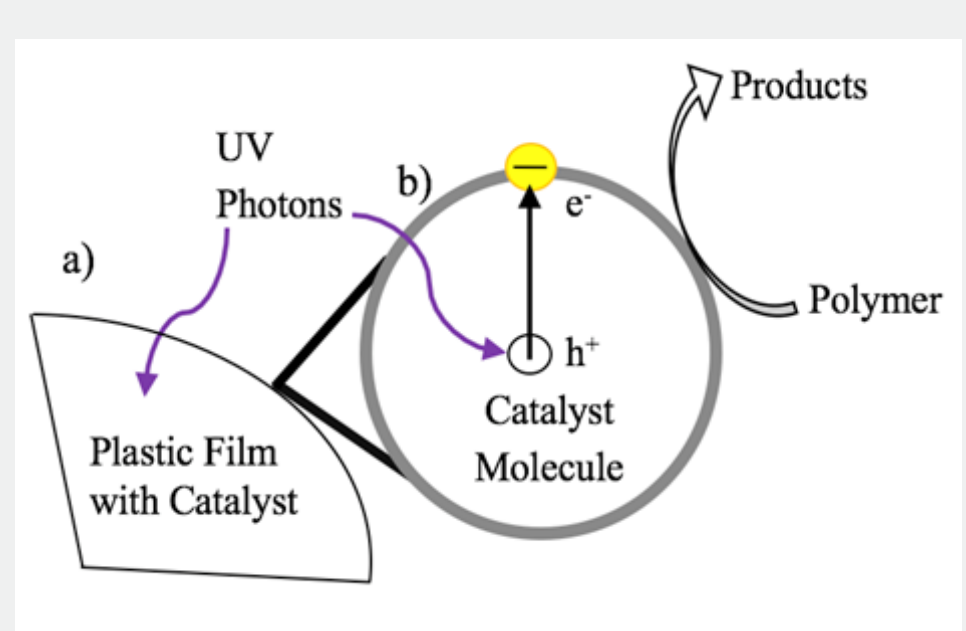

Figure 1: (a) Schematic depicting the macroscopic and microscopic (b) photocatalytic mechanisms utilized to sustain polymeric decay.

\section{Experimental}

\section{Materials}

The three pure-grade plastics (HDPE, LDPE, PS) were purchased from Sigma Aldrich (St. Louis, MO) and PET resin was purchased from Eastman Chemical Company (Kingsport, TN). The nanoparticle-sized photocatalysts employed were: anatase $\mathrm{TiO}_{2}$ $\mathrm{ZnO}, \mathrm{WO}_{3}$, and $\mathrm{Fe}_{2} \mathrm{O}_{3}$ with d50 particle diameter values of $25 \mathrm{~nm}$, $50 \mathrm{~nm}, 100 \mathrm{~nm}$ and $100 \mathrm{~nm}$, respectively. The sizes of these oxide additives were at least 3 orders of magnitude smaller than the thicknesses of the fabricated test films. Catalysts were purchased from Sigma Aldrich (St. Louis, M0). The photocatalysts were mixed with the polymers and exposed to UV in aluminum weigh dishes. Two sets of each polymer were made with up to $6 \% \mathrm{wt}$ photocatalysts, as well as a photocatalyst-free control. The lights employed were ultraviolet-A (UVA), ultraviolet-B (UVB), and ultraviolet-C (UVC), with wavelengths of 400-315 nm, 315-280 $\mathrm{nm}$, and 280-100 $\mathrm{nm}$, respectively. The photocatalysts examined are active for UV wavelengths from $290 \mathrm{~nm}$ to $400 \mathrm{~nm}$ and have band gaps of 3.2, 3.37, 2.6, and $2.1 \mathrm{eV}$ for $\mathrm{TiO}_{2}, \mathrm{ZnO}$, and $\mathrm{WO}_{3}$, and $\mathrm{Fe}_{2} \mathrm{O}_{3}$, respectively. The band gap corresponds to the energy required to create a free electron and a hole in the catalyst. The exciton then interacts selectively with the carbon backbone and can initiate decay at specific energies generally with photons 
in the UV spectra. As band-gap increases, the below-gap losses prohibit effective photo decay whereas the exciton pairs from a low band-gap catalyst are not sufficiently energetic to initiate decay. Experimentation was conducted in two parts as described below. One part examined environmental conditions that may inhibit decomposition and the other examined product-analogs as an industrial scalability analysis.

\section{Sample Formation}

\section{Environmental Effects Samples}

The plastics were melted in the aluminum weigh dishes using a QL Model 40GC laboratory oven and hot plate based on melting temperature. Polyethylene terephthalate, for example, has a high melting temperature of about $240{ }^{\circ} \mathrm{C}$ and was melted using the direct heat of a hot plate [19]. Low-density polyethylene, HDPE, and PS were melted in the lab oven at approximately $200{ }^{\circ} \mathrm{C}$. The photocatalyst was integrated into the leathery-state polymers using a metal rod and stirred for five minutes. The plastics were allowed to set for approximately one day at room temperature and then placed in the light exposure unit.

\section{Catalyzed Polymer Film Formation}

Although the overall measurement of photocatalytic decomposition was the same in the environmental conditions study and film photodecay study, there were several key procedural modifications and improvements represented. Each plastic (PET, HDPE, LDPE, PS) was weighed with the respective photocatalysts for new samples. The addition of Fe203 allowed for a different photocatalyst band gap to be studied in the catalyzed films. To simulate real-world plastic formation with minimal oxidation and to better mix the catalysts with the polymers, a heat-press was used to form the samples. The plastics were made into films with thicknesses averaging $0.55 \mathrm{~mm}$ with a standard deviation of 0.12 $\mathrm{mm}$. The plastics were mixed with the catalyst in the aluminum weigh dishes and transferred to Kapton ${ }^{\circledR}$ film, a heat-resistant polymer used in heat-pressing. Each $5 \mathrm{~cm}$ diameter sample was pressed twice with under one ton of force on a Carver heat press. The temperature for the heated plates was chosen based on the melting and glass transition points of the polymers. The plastics (PET, HDPE, LDPE, and PS) were melted at $240{ }^{\circ} \mathrm{C}, 140{ }^{\circ} \mathrm{C}, 190$ ${ }^{\circ} \mathrm{C}$, and $210{ }^{\circ} \mathrm{C}$, respectively. Following the first pressing, the film was cut into sections and pressed again. By cutting and repressing the plastics, the catalyst was integrated evenly into the structure of the plastics. The cooled films were then cut into samples for tensile strength analysis and UV exposure.

\section{UV Exposure}

\section{The Effects of Water and UV Wavelength on Accelerated Thermoplastic Photodecomposition}

A UV-secure testing hood was used to house the experiments. Two UVA bulbs and one UVC bulb were attached to the top of the hood with wattages of $32 \mathrm{~W}$ and $16 \mathrm{~W}$, respectively. The $20 \mathrm{~W}$ UVB lamp was placed in a standard, lab-clamp socket and clamped to a ring stand as shown in the supplemental data, Figure 1a. A 3.2\% salt solution, to simulate the median salinity of ocean water, and distilled water were used to simulate the effect of submersion on the plastic decomposition in salt and fresh water. Two sets of each plastic were made, so each plastic could be tested in salt water and freshwater conditions. Two control dishes, one containing only salt water and one containing only fresh water, underwent the same conditions as the plastic samples to determine any weight loss in the dish without a polymer. Water was removed for the last two weeks to determine if the plastics continued to lose weight in the different environments. A Vernier LabQuest UVB probe (Beaverton, OR) was used throughout experimentation to monitor the UV conditions inside the hood and to determine any changes in exposure rate. The samples were exposed to UV light for eight weeks. Every seven days, the samples were washed with distilled water, dried in a drying oven, and weighed. Weighing the samples after washing the dishes allowed for the removal of any salt buildup and possible decomposition products. Percent weight loss over time was used to quantify the release of decomposition products. The samples were resubmerged in water following each weighing. Rotation was conducted on average every two to four days to avoid uneven decomposition due to differences in distances the samples were from the light sources. Water was replenished during rotation to maintain sample submersion.

\section{Photodecomposition of Catalyzed Polymer Films Sample Formation}

The samples were placed in aluminum weigh dishes and exposed to UVA and UVB without water. Control dishes, plastics without catalysts, and catalyzed plastics outside the UV apparatus were used for comparative purposes. The samples were washed, dried, and weighed every seven days. The amount of UVB was at $50 \%$ for the first three weeks and $100 \%$ for the rest of experimentation. The exposure apparatus was constructed with two 32 W UVA bulbs and two 15 W UVB bulbs. Aluminum flashing was used to provide optimal reflection in the apparatus. The samples were rotated every two to four days on average. The samples were exposed for an eight-week trial period. The samples with the highest weight loss were chosen for analysis with higher catalyst concentration. Six additional samples, two sets of HDPE and LDPE with a $4 \%$ and $6 \%$ concentration of $\mathrm{TiO}_{2}$ catalyst, were pressed similarly to the $2 \%$ samples. Tensile strength testing was conducted on HDPE and LDPE control, $2 \%, 4 \%$, and $6 \%$ catalyst concentrations. The samples were cut into tensile dog-bone shape for testing using a 3-D printed template. Three plastic specimens were made for each plastic and catalyst concentration to total 24 tensile specimens. The HDPE and LDPE plastics were chosen to compare the various catalyst concentrations on the tensile strength. The gauge width, gauge length, and thickness were measured using digital calipers for tensile strain calculations. An Instron Model 5867 electromechanical test frame (Canton, MA) was used with a $1000 \mathrm{~N}$ load cell and 80 psi pneumatic film tensile 
grips. A crosshead displacement rate of $1 \mathrm{~mm} / \mathrm{min}$ was used for the tensile testing. The breaking force was used to calculate stress.

$$
\sigma=F\left(G^{*} T_{s}\right)^{-1}
$$

where $\sigma$ is the tensile stress in $\mathrm{MPa}, \mathrm{F}$ is force in $\mathrm{N}, \mathrm{GW}$ is gauge width of the cut shape in $\mathrm{mm}$, and Ts is thickness of each plastic film in mm. 2.4

\section{Sample Analysis}

To compare the enhanced decomposition with photocatalysts to frequently used materials, the plastic thickness lost on the top layer over time was calculated and used to approximate a simulated time span of household products for real-world use. The decay approximation was conducted in order to normalize the results from these experiments with standard plastics handled by consumers. Each plastic was paired with a commonly littered plastic counterpart. Polyethylene terephthalate plastic bottles, HDPE milk jugs, LDPE plastic produce bags, and PS disposable eating utensils were cut into rectangles and measured with calipers to determine the thicknesses shown in Table 1. Thickness loss, $\mathrm{T}_{\mathrm{L}}$ in $\mathrm{cm}$, was determined by

$$
T_{L}=\frac{V}{A}
$$

where $\mathrm{A}$ is the area of the samples in $\mathrm{cm}^{2}$, and $\mathrm{V}$ is the volume of the samples solved for by dividing the change in weight of the samples by the density of the plastics. The equation:

$$
\frac{T_{L}}{8}=\frac{T_{R}}{x}
$$

models the theoretical time in weeks for full decomposition, $\mathrm{x}$, where $\mathrm{T}_{\mathrm{L}}$ is divided by eight weeks and $T_{R}$ is the thickness of household plastics in Table 1.

Table 1: Household Plastic Data.

\begin{tabular}{|c|c|c|c|}
\hline Polymer & Common Use & $\begin{array}{c}\text { Thickness of Household Materi- } \\
\text { als (mm) }\end{array}$ & Density of Polymer $\mathbf{( g / \mathbf { c m } ^ { 3 } )}$ \\
\hline PET & Plastic Bottle & 0.15 & 1.39 \\
\hline HDPE & Milk Jug & 0.4 & 0.947 \\
\hline LDPE & Plastic Bag & 0.02 & 0.918 \\
\hline PS & Plastic Utensil & 0.6 & 0.971 \\
\hline
\end{tabular}

\section{Results and Discussion}

\section{Effects of Water and Wavelength on Polymer Photodecomposition}

Polymers were selected due to their brief application lifetime in a specific task. For example, many plastics such as food packaging and grocery bags are only used for a short period of time. Additionally, the smallest possible particle size available was used to maximize surface area and thus increase the photocatalytic activity [20]. Anatase $\mathrm{TiO}_{2}$ was used due to a different crystalline configuration in comparison to rutile $\mathrm{TiO}_{2}$ since anatase $\mathrm{TiO}_{2}$ produces longer-lived free electrons which can interact with the plastics more directly [21]. The UV light that passes through the ozone layer lies in UVA and UVB ranges; UVC was also employed in some instances due to its ability to accelerate the photocatalytic decomposition of plastics and the long timeline of experimentation [22]. The supplemental data shows the percent weight loss for the samples for fresh and salt water of PET, HDPE, LDPE, and PS, respectively. Over the eight-week period, plastics integrated with the $\mathrm{WO}_{3}$ catalyst experienced more weight loss than the other catalysts in each material except PET. The $\mathrm{TiO}_{2}$ samples had the second-highest weight loss followed by $\mathrm{ZnO}$. Samples in salt water generally showed higher weight loss than those in fresh water. This environmental condition study was used as a proof of concept for the production of scalable polymer laminates embedded with photocatalysts capable of initiating and sustaining decay.

\section{Film Accelerated Photo decay and Modern Product Comparison}

The percent weight lost in the HDPE and LDPE samples, respectively, are shown in Figure 2a \& $2 \mathrm{~b}$. Tungsten oxide and iron oxide catalyst results were not included in Figure $2 \mathrm{a} \& 2 \mathrm{~b}$ because the exposure treatment caused insignificant weight loss over the eight-week trial period. The bars represent the average of the two samples for each plastic. The trend lines' values representing the percent weight loss for samples one and two with the $\mathrm{TiO}_{2}$ catalyst in HDPE and LDPE over the eight-week period had $\mathrm{R}^{2}$ values of 0.982 and 0.996 for HDPE and 0.991 and 0.969 for LDPE. Using an HDPE milk jug and LDPE plastic bag as real-world analogs for the thickness lost in experimentation, the extrapolated time to total thickness degradation is depicted in Figure $2 \mathrm{c} \& 2 \mathrm{~d}$. The theoretical time for full decay of a HDPE milk jug is shown in Figure 2c, and the theoretical time for full decay of a LDPE plastic bag is shown in Figure 2d. Both of these extrapolative measurements assumed linear film loss across the UV exposed surface with a $2 \%$ catalyst load. A sample with a zero-weight change was equated to the average of the control. The plastics with the greater weight loss were modeled over time in Figure 3. The average decomposition 
of HDPE with $4 \%$ and 6\% TiO2 samples is shown in Figure 3a, and the average decomposition of LDPE with $4 \%$ and $6 \%$ TiO2 samples is shown in Figure $3 \mathrm{~b}$. The $\mathrm{R}^{2}$ values for Figure $3 \mathrm{c}$ were
0.983 and 0.988 for HDPE $4 \%$ and $6 \%$, respectively. The $4 \%$ and $6 \% \mathrm{R}^{2}$ values for Figure $3 \mathrm{~b}$ were 0.995 and 0.992 for LDPE, respectively.
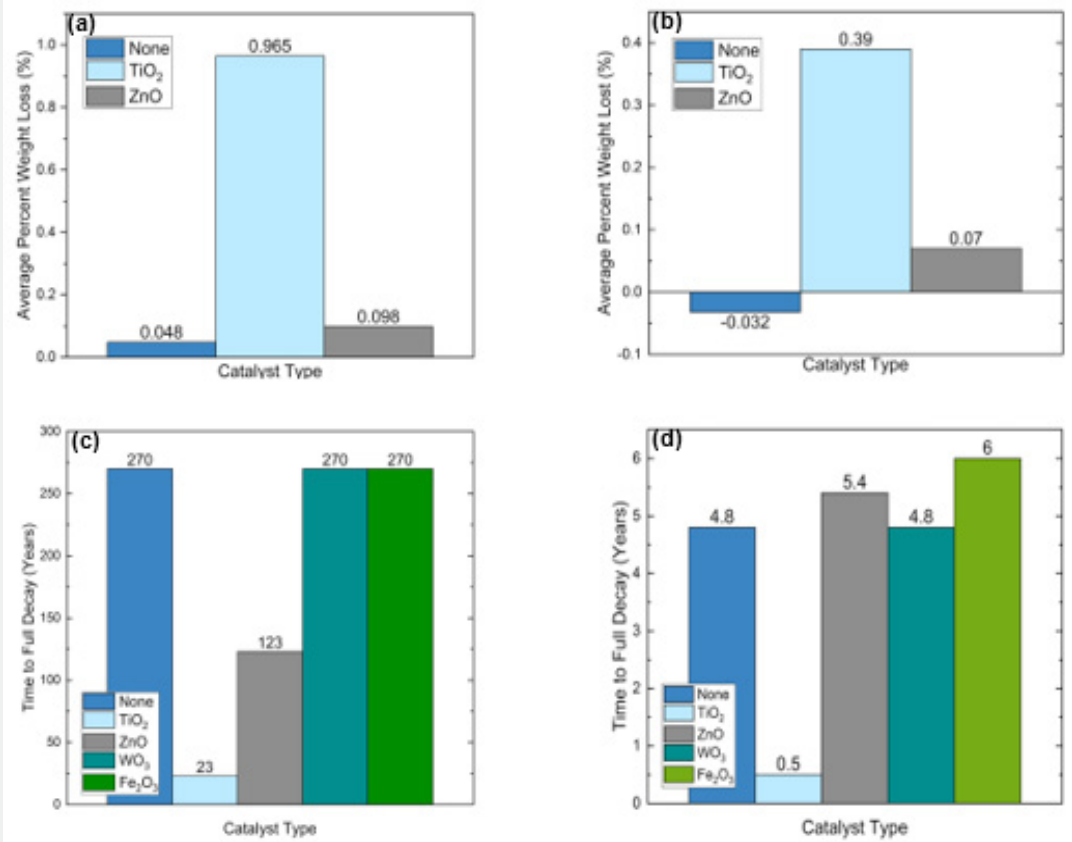

Figure 2: (a) The average percent weight lost after exposure to simulated UV and visible light environment of HDPE (b) LDPE (c)An extrapolated theoretical decay of an HDPE milk jug "analog" made with similar thickness and a $2 \mathrm{wt} \%$ load of catalyst and (d) A plastic shopping bag was used as the LDPE analog for comparing the decay LDPE with and without various catalysts.
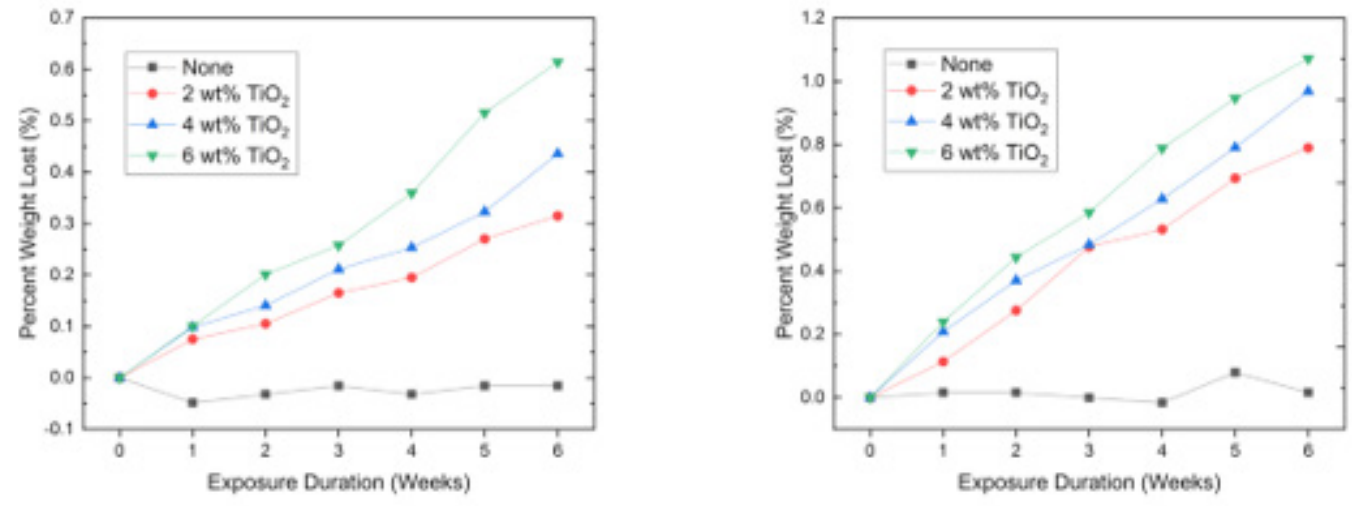

Figure 3: (a)The total decay trend of various catalyst concentrations of anatase TiO2 embedded in laminates of HDPE (b) LDPE (c) Increasing the catalyst/polymer ratio affected the rate of decomposition compared to the control.

\section{Tensile Strength Testing}

Tensile strength testing was used to determine how the addition of catalysts affected the strength and the potential usefulness of the plastics. The tensile strength curves of HDPE and LDPE samples that have not been exposed to UV light are shown in Figure 4. Tensile strength was measured as stress in MPa for given displacements of the film tensile grips in $\mathrm{mm}$. The maximum tensile stress/tensile strength was determined for each concentration of catalyst $(0 \mathrm{wt} \%, 2 \mathrm{wt} \%, 4 \mathrm{wt} \%$, and 6 $\mathrm{wt} \%)$. The tensile strengths for the respective plastic and catalyst 
specimen were averaged and represent highest point of stress before the sample deformed or cracked in the testing apparatus. The addition of $\mathrm{TiO}_{2}$ did not drastically affect the stress capacity of the samples. The averages of each maxima of the three HDPE and LDPE tensile specimens are shown in Figure 4. Scanning electron microscope (SEM) images were taken using a Zeiss Merlin SEM
(Oberkochen, Germany). The images were taken before and after UV exposure. The HDPE with $2 \mathrm{wt} \% \mathrm{TiO}_{2}$ before UV exposure is shown in Figure 5a and the same sample after exposure is shown in Figure 5b. The images were used to confirm the photo-driven surface modifications following the UV exposure.
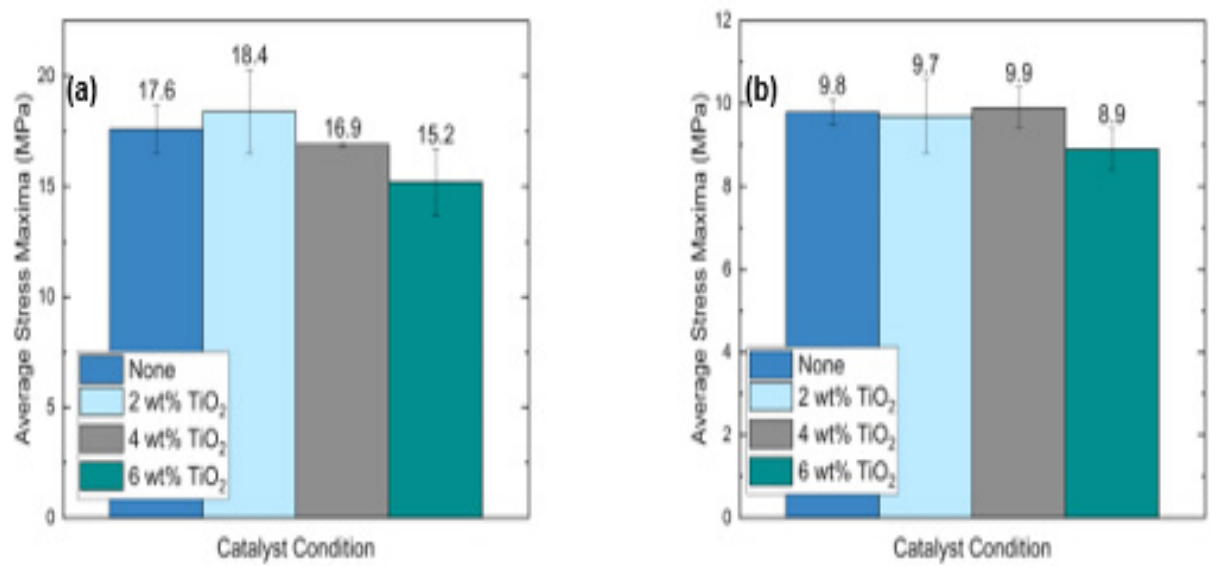

Figure 4: (a) The tensile maxima of varying catalyst concentrations in HDPE (b) in LDPE.

\section{Variations in Weight Loss Over Simulated Environmental Conditions in the Environmental Conditions Study}

Samples loaded with photocatalysts exhibited greater weight loss than the control samples, confirming the conjecture that photocatalysts can accelerate polymer decay when submerged in both salt and fresh water as a simulation of lake, rivers, and oceanic environments where plastic litter is commonly observed. Differences between salt and fresh water were present in the samples. Salt water has a higher index of refraction than fresh water which causes the water to have a different critical angle in the transfer of the UV light through the water to the plastic. The impact of the salinity on the plastic structure was not known, but the combination of a higher index of refraction of salt water on materials may have increased the effectiveness of the salt solution. The water did block a portion of the light; however, most plastics float based on their lower density which would allow for a greater likelihood of direct exposure to sunlight in the environment. The photocatalyst integration used in this primary environmental condition study was not ideal and prompted adjustments for the second set of experiments with film photodecomposition. The photocatalysts in the environmental test may have not been thoroughly embedded or integrated to provide optimal decomposition in the UV environment.

The difference in the weight loss of PET and the other plastics is consistent with the different shapes and configurations of the molecular structures of the polymers and the decay sequence of each polymer. Weight loss did not consider changes in chemical structure other than the escape of airborne or surfacedecomposition products. PET had relatively no weight loss with $\mathrm{WO}_{3}$ as illustrated in supplemental Figure 2 while the largest weight changes were with the $\mathrm{WO}_{3}$ for HDPE, LDPE, and PS, respectively. An unexpected result was that the WO3 PET and control HDPE gained weight. The increase in weight may have been the result of the catalyst molecules and polymers attracting and retaining moisture. Additionally, during the drying process, some moisture could have remained in the microscopic structure of the plastic or catalyst. The catalyst molecules, especially $\mathrm{TiO}_{2}$, can retain water due to an inherent characteristic of some highly porous crystalline solids [23]. Additionally, UVC is more energetic and can increase oxidative/radical decomposition process [24] during exposure based on the polymer and catalyst absorption spectrum. Earth's ozone layer and other atmospheric gases filter out UVC which results in UVA and UVB comprising $95 \%$ and $5 \%$ of incident UV light at Earth's surface, respectively [25]. Thus, the UVC in the experiments was less representative of actual decomposition expected in the environmental conditions. Additionally, concerns remain regarding the effect of UVC on experimentation to yield unrealistic results for scalable applications by stimulating different photocatalytic band gaps than energies present in the solar spectrum incident on Earth's surface. These concerns were addressed in the second set of experiments involved thermal laminates with the catalyst integrated into films for photodecay. 


\section{Analysis of UV Stimulated Photodecomposition of the Polymer films}

The techniques employed in the film experiments illustrate that photocatalysts can be added to plastics during the recycling or industrial formation process. The ability to successfully heatpress the samples illustrates the viability for the use of heatpressing and injection molding after photocatalyst integration in industry. All of the photocatalysts were easily and readily incorporated into the polymers with no discoloration or oxidation even though the loading was as high as $6 \%$ wt. The decay of the four plastics in films illustrates a successful comparison to real-world application of photocatalyst integration in products. HDPE and LDPE, respectively, shown in Figure 2a and 2b, show that the samples with $\mathrm{TiO}_{2}$ experienced the greatest weight loss. The weight change in HDPE and LDPE is attributed to the decay mechanism of polyolefins, while PET and PS decompose to nongaseous radicals and oxygenates. Photocatalysts produce radicals in HDPE and LDPE which break the polymer backbone and cause the release of ethylene gas and other gaseous byproducts. Additionally, the $\mathrm{R}^{2}$ values close to one for the decay trends of HDPE and LDPE illustrate a consistent linear decay seen over the eight-week trial period. The insignificant weight loss seen in PET and PS demonstrates these polymers have different modes of decomposition by UVA and UVB light when integrated with catalyst molecules in comparison to HDPE and LDPE. One explanation for the insignificant weight loss in the PET and PS samples may be that the $\mathrm{TiO}_{2}$ catalyst was absorbing the UV light rather than initiating decay.

The differences in UV activity among the various polymer molecules demonstrate evidence for the varying degrees of decomposition seen during experimentation. Polyethylene terephthalate decays in a process of chain severing where large organic polymer chains break into smaller organic chains. In contrast, polyethylene exhibits decay into gaseous ethylene monomers [5]. The examined samples had evident color change, cracking, and surface delamination. The weight loss of PET and PS illustrated similar life spans between the control and the catalyzed samples. HDPE and LDPE decay, respectively, shown in Figure 2c \& 2d, had significant decreases in weight in the $\mathrm{TiO}_{2}$ samples. For an HDPE milk jug, the $\mathrm{TiO}_{2}$ sample had a decay that was approximately $91.48 \%$ faster than the control. Thus, a milk jug would decay in about 270 years without a catalyst and about 23 years with the $\mathrm{TiO}_{2}$ catalyst. Similarly, a LDPE plastic bag with $\mathrm{TiO}_{2}$ theoretically would decay approximately $89.58 \%$ faster than the control samples. A bag would decay in approximately 4.8 years without a catalyst and about 0.5 years with the $\mathrm{TiO}_{2}$ catalyst.

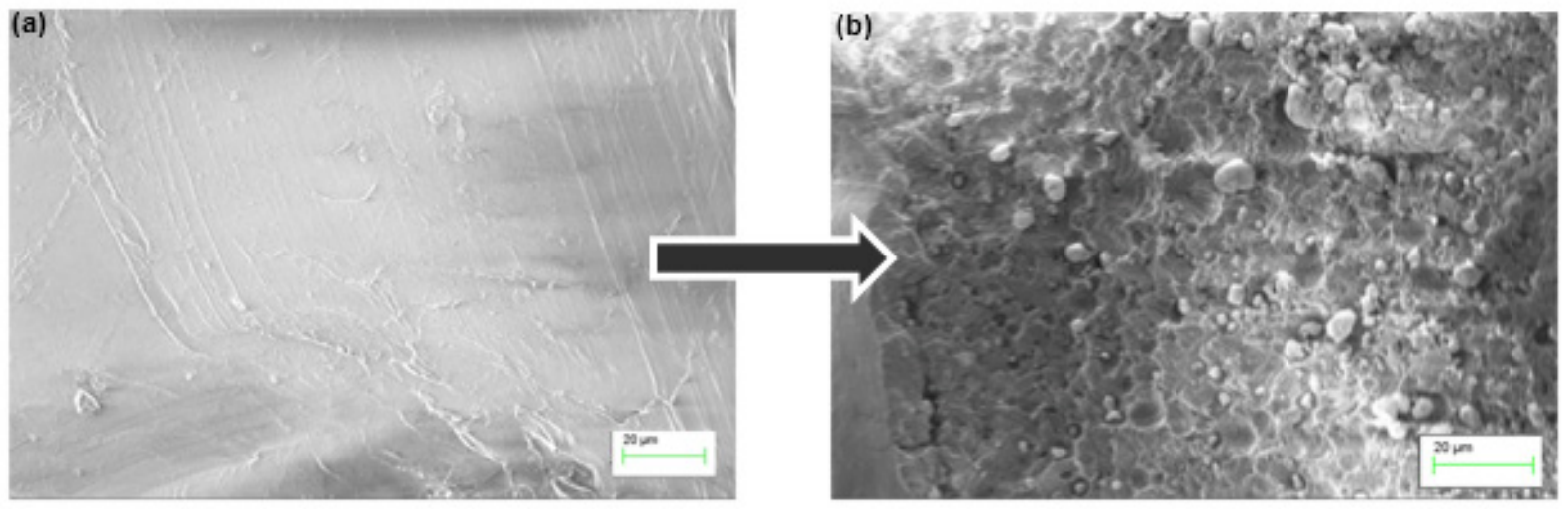

Figure 5 : (a) An HDPE sample with a 2 wt\% catalyst concentration before UV/visible exposure and (b) following the continuous light exposure.

The HDPE and LDPE $4 \%$ and $6 \% \mathrm{TiO}_{2}$ samples exposed to UVA and UVB are displayed in Figure 3. Over the six-week exposure period, the $6 \%$ samples decayed approximately $50 \%$ faster than the $2 \%$ samples. The further accelerated decay illustrates that increasing the amount of catalyst in the plastic affects the decomposition of the plastic and demonstrates that varying the concentration of the catalyst could be used in industry to produce desired decomposition periods for their products. Moreover, the $\mathrm{R}^{2}$ values close to one illustrate the linear nature of HDPE and LDPE decay through weight loss. The tensile strength data were used to determine whether the addition of catalyst would prevent the daily use of common items due to an inability of the polymers to withstand forces. HDPE and LDPE, with concentrations of $0 \%, 2 \%, 4 \%$ and $6 \%$, maintain their resistance to stress with the addition of the catalyst as shown in Figure 4. The HDPE specimen with $4 \%$ concentration had a $4 \%$ decrease and the $6 \%$ concentration had a $14 \%$ decrease in the average maximum stress compared to the control. The LDPE specimen maintained tensile strength up to a $6 \%$ concentration in which the maxima average decreased $10 \%$ compared to the control. The differences 
in stress vs. displacement represent a slight decrease in the strength of the HDPE and LDPE with the addition of the catalyst at higher percentages, but insignificant stress changes among the lower percentage catalyst samples. The SEM images further reinforce evidence of decomposition. Within the polymer matrix, surface features as well as evident macroscopic delamination on the plastic surface is shown in Figure 5a while a smooth polymer surface studded with the $\mathrm{TiO}_{2}$ photocatalyst particles is shown in Figure $5 b$

\section{Analysis of Surface Area and Porosity on Catalyst Efficacy}

Negative weight loss was attributed to water absorption within the polymer matrices and catalyst structures. Catalysts were also compared using percent weight loss per area catalyst and volume catalyst to determine the interactive effects of density and catalyst porosity on the rate of decay. High surface area interaction of the catalyst with the surrounding laminate polymer accelerated decomposition by allowing greater ease of maximized energy exciton interaction. Particle sizes of the catalysts were estimated using nitrogen gas adsorption (Brun Auer-Emmett-Teller (BET)) measurements on a micrometrics TriStar II Plus Physisorption Analyzer (Norcross, GA), and their surface areas are shown in Table 2 . The samples were heated at $300^{\circ} \mathrm{C}$ for 60 minutes before BET measurements were taken. Figure 6 illustrates the effects of various catalysts on the mass loss per unit catalyst area and volume.
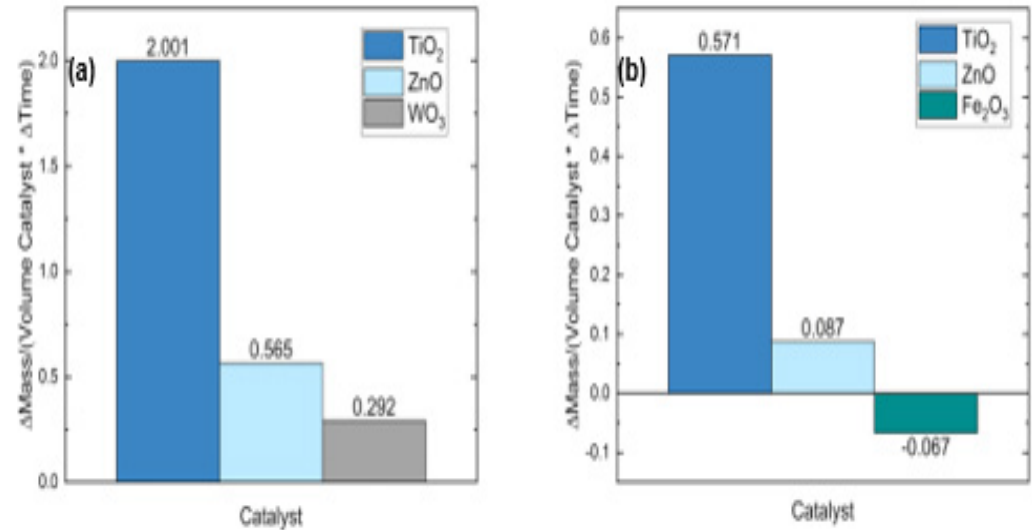

Figure 6: (a) The relation of the catalyst's volume to the mass loss rate in HDPE and (b) LDPE for the polymer films with given decomposition parameters. Fe2O3 in HDPE and WO3 in LDPE exhibited no significant mass loss over the course of the exposure period.

Table 2: Surface area of photocatalysts.

\begin{tabular}{|c|c|c|}
\hline Catalyst & BET Surface Area $\left(\mathrm{m}^{2} / \mathrm{g}\right)$ & Error $\pm(\%)$ \\
\hline $\mathrm{TiO}_{2}$ & 50.01 & 0.37 \\
\hline $\mathrm{WO}_{3}$ & 7.59 & 0.3 \\
\hline $\mathrm{ZnO}$ & 30.16 & 0.19 \\
\hline $\mathrm{Fe}_{2} \mathrm{O}_{3}$ & 30.73 & 0.87 \\
\hline
\end{tabular}

Among the highest weight lost HDPE and LDPE samples, $\mathrm{TiO}_{2}$ did have an advantage due to a higher surface contact area. Zinc oxide, at the same weight percent, interacted about a quarter as much as $\mathrm{TiO}_{2}$. As a result, a higher volume concentration of zinc could have similar decomposition effects as $\mathrm{TiO}_{2}$. Novel catalysts such as porous gamma alumina could be coated in a catalyst to further enhance decomposition by increasing catalyst surface area. Some samples exhibited negative change in mass attributed to water absorption in the catalyst or polymer matrix. Furthermore, PET and PS exhibited minimal mass changes that are insignificant in comparison to the original mass and the mass lost by HDPE and LDPE. Noting the change in conditions between the environmental conditions study and film photodecay study, the use of $\mathrm{TiO}_{2}$ poses the best characteristics for worldwide application of photocatalysts to protect the environment from plastic litter. The end products of the decomposition can be integrated back into the carbon and water cycles. By decomposition of polluted plastics, the plastics' hydrocarbon backbone can be returned to the carbon cycle to eventually produce more plastics in the future.

\section{Conclusion}

Given the rampant growth of polymer litter affecting Earth's oceans, our experiments outline a scalable and cost-effective means to mitigate the environmental impacts of commonly littered plastic products. The environmental condition portion of this work illustrates the applicability of photocatalytic decomposition as a means to control plastic litter since polymers could effectively decay both in high energy UVC environments but also in the presence of UVA/UVB light which can penetrate the atmosphere. Moreover, the impact of water submersion in both salt and fresh water illustrates that decomposition could be maintained in common environmental sinks for littered plastic products. The film photodecay study outlines the scalability and general utility of polymers embedded with photocatalytic particles and illustrates that neither the initial tensile strength of the plastic 
nor its production methods would need to be drastically altered to introduce photocatalysts. In addition, photocatalysts can be added to plastics to enhance the decay in the environment without the need for manipulating the chemical makeup of the precursor polymer or sacrificing the usefulness of the plastics. The plastics also are not significantly affected with respect to cost. Given the nature of the research-grade materials used in this investigation, the catalyst additives as well as the production parameters could be optimized to maximize profit while minimizing the litter/waste footprint. While both $\mathrm{TiO}_{2}$ and $\mathrm{ZnO}$ were both effective in the UVA/UVB simulated conditions, the anatase $\mathrm{TiO}_{2}$ particles acted to significantly reduce the longevity of the HDPE and LDPE films. The $\mathrm{TiO}_{2}$ catalyst also acted to enhance tensile strength in low concentrations (below $4 \mathrm{wt} \%$ in HDPE and below $6 \mathrm{wt} \%$ in LDPE) and maintains a low cost due to the prolific use of titania in a wide scope of products. Thus, the addition of a catalyst, especially anatase $\mathrm{TiO} 2$, would be a scalable and economical protection mechanism against plastic litter by sustaining photodecay under ambient solar conditions.

\section{Acknowledgement}

The authors would like to thank Jessica Williams, Tammy Carneim, Deanna Pickel, and Sharon Thomas of Oak Ridge High School in Oak Ridge, TN for their support of student research initiatives. The authors would also like to thank Richard Pagni, Gajanan Bhat, Stephen Sheriff of the University of Tennessee, Knoxville, and Beth Armstrong, Alicia Mayville and Shirley Waters of Oak Ridge National Laboratory. This research was conducted in coordination with resources at the University of Tennessee, Knoxville Nonwovens Materials Research Laboratory and Oak Ridge National Laboratory's Materials Demonstration Facility/National Transportation Research Facility. Funding was provided by the Southern Appalachian Science and Engineering Fair Teacher Grant. This manuscript has been authored by UTBattelle, LLC, under contract DE-AC05-000R22725 with the US Department of Energy (DOE).

\section{References}

1. Cole M, Lindeque P, Halsband C, Galloway TS (2011) Microplastics as contaminates in the marine environment: a review. Mar Pollut Bull 62(12): 2588-2597.

2. Erikson M, Lebreton LCM, Henry S, Charles JM, Jose CB, et al. (2015) Plastic Pollution in the World's Oceans: More than 5 Million Plastic Pieces Weighing over 250,000 Tons Afloat at Sea, PLoS ONE 9(12): $1-15$.

3. (2015) US National Park Service, Time It Takes for Garbage to Decompose in the Environment.

4. Chanda M, Roy SK (2007) Plastics Technology Handbook, CRC Press, Baton Roca, USA.

5. Zweifel H (2001) Plastics Additives Handbook. $5^{\text {th }}$ ed $^{\text {n }}$, Hanser Gardner, Cincinnati, USA.

6. Guillet JE (1972) Fundamental Processes in the UV Degradation and Stabilization of Polymers. Pur Appl Chem 30(1-2): 135-44.
7. Manangan T, Shawaphun S (2010) Nano-Sized Titanium Dioxides as Photo-Catalysts in Degradation of Polyethylene and Polypropylene Packagings. Science Journal Ubon Ratchathani University 1(2): 14-20.

8. Zhao Xu, Li Z, Liyi S (2007) Solid-phase photocatalytic degradation of polyethylene plastic under UV and solar light irradiation, J Mol Catal A: Chem 268(1-2): 101-106.

9. Ali SS, Qazi IA, Muhammad A, Zahiruddin K, Thomas C, et al. (2016) Photocatalytic degradation of low-density polyethylene (LDPE) films using titania nanotubes. Environmental Nanotechnology, Monitoring \& Management 5: 44-53.

10. Thomas RT, Nair V, Sandhyarani N (2013) $\mathrm{TiO}_{2}$ nanoparticle assisted solid phase photocatalytic degradation of polythene film: A mechanistic investigation, Colloids Surf, A 422(5): 1-9.

11. Hashimoto $\mathrm{K}$, Irie $\mathrm{H}$, Fujishima $\mathrm{A}$ (2005) $\mathrm{TiO}_{2}$ Photocatalysis: A Historical Overview and Future Prospects. Jpn J Appl Phys 44(12): 8269-8285.

12. Gupta SM, Tripathi M (2011) A review of $\mathrm{TiO}_{2}$ Nanoparticles. Chin Sci Bull 56: 1639.

13. Weisner MR, Lowry GV, Biswas P, Pedro A, Dionysiou D (2006) Assessing the Risks of Manufactured Nanomaterials. Environ Sci Technol 40(14): 4336-4445.

14. Shi H, Magaye R, Castranova V, Zhao J (2013) Titanium Dioxide Nanoparticles: A Review of Current Toxicological Data. Particle and Fibre Toxicology 10: 15 .

15. Fu G, Vary PS, Lin CT (2005) Anatase $\mathrm{TiO}_{2}$ Nanocomposites for Antimicrobial Coatings. J Phys Chem B 109(18): 8889-8898.

16. Hara-Kudo K, Segawa Y, Kimura K (2006) Sanitation of seawater effluent from seaweed processing plants using a photo-catalytic $\mathrm{TiO}_{2}$ oxidation, Chemosphere 62(1): 149-154.

17. Sirsat S, Neal J (2015) Titanium Dioxide Nanoparticles as an Environmental Sanitizing Agent, J Microb Biochem Technol 7: 61-64.

18. Weir A, Westerhoff P, Lars F, Goetz N (2012) Titanium Dioxide Nanoparticles in Food and Personal Care Products. Environ Sci Technol 46(4): 2242-2250.

19. Brydson JA (1995) Plastics Materials. $6^{\text {th }} \mathrm{ed}^{\text {n }}$, Butterworth-Heinemann, Boston, USA.

20. Xu N, Shi Z, et al. (1999) Effects of Particle Size of $\mathrm{TiO}_{2}$ on Photocatalytic Degradation of Methylene Blue in Aqueous Suspensions, Ind Eng Chem 38(2): 373-379.

21. Zhang J, Zhou P, Liu J, Yu J (2014) New Understanding of the Difference of Photocatalytic Activity among Anatase, Rutile and Brookite $\mathrm{TiO}_{2}$, Phys Chem Chem Phys 16(38): 20382-20386.

22. Gijsman P, Meijers G, Vitarelli G (1999) Comparison of the UVDegradation Chemistry of Polypropylene, Polyethylene, Polyamide 6 and Polybutylene Terephthalate, Polym Degrad and Stab 62(3): 433441.

23. Voorde M, Werner M, Fecht H (2004) The Nano-Micro Interface: Bridging the Micro and Nano Worlds, Wiley-VCH, Weinheim, Germany.

24. Yousif E, Haddad R (2013) Photodegradation and photostabilization of polymers, especially polystyrene: review. SpringerPlus 2(1): 1-32.

25. (2015) US Department of Health and Human Services. Scientific Review of Ultraviolet (UV) Radiation, Broad Spectrum and UVA, UVB, and UVC. 
(C) (i) This work is licensed under Creative BY DOI: 10.19080/AJOP.2020.04.555633
Your next submission with Juniper Publishers will reach you the below assets

- Quality Editorial service

- Swift Peer Review

- Reprints availability

- E-prints Service

- Manuscript Podcast for convenient understanding

- Global attainment for your research

- Manuscript accessibility in different formats

( Pdf, E-pub, Full Text, Audio)

- Unceasing customer service

Track the below URL for one-step submission https://juniperpublishers.com/online-submission.php 